\title{
Intelligent Fuzzy Control with State-Derivative Feedback for Takagi-Sugeno Fuzzy Stochastic Singular Systems
}

\author{
Wen-Jer Chang \\ Department of Marine Engineering, National Taiwan Ocean University, Keelung, Taiwan, R. O. C., \\ wjchang@mail.ntou.edu.tw \\ Kuang-Yow Lian \\ Department of Electrical Engineering National Taipei University of Technology Taipei, Taiwan, R.O.C., \\ kylian@mail.ntut.edu.tw \\ Cheung-Chieh Ku \\ Department of Marine Engineering, National Taiwan Ocean University, Keelung, Taiwan, R. O. C. \\ Che-Lun Su \\ epartment of Marine Engineering, National Taiwan Ocean University, Keelung, Taiwan, R. O. C.
}

Follow this and additional works at: https://jmstt.ntou.edu.tw/journal

Part of the Fresh Water Studies Commons, Marine Biology Commons, Ocean Engineering Commons, Oceanography Commons, and the Other Oceanography and Atmospheric Sciences and Meteorology Commons

\section{Recommended Citation}

Chang, Wen-Jer; Lian, Kuang-Yow; Ku, Cheung-Chieh; and Su, Che-Lun (2021) "Intelligent Fuzzy Control with StateDerivative Feedback for Takagi-Sugeno Fuzzy Stochastic Singular Systems," Journal of Marine Science and Technology: Vol. 29: Iss. 3, Article 6.

DOI: $10.51400 / 2709-6998.1435$

Available at: https://jmstt.ntou.edu.tw/journal/vol29/iss3/6

This Research Article is brought to you for free and open access by Journal of Marine Science and Technology. It has been accepted for inclusion in Journal of Marine Science and Technology by an authorized editor of Journal of Marine Science and Technology. 


\title{
Intelligent Fuzzy Control with State-Derivative Feedback for Takagi-Sugeno Fuzzy Stochastic Singular Systems
}

\author{
Wen-Jer Chang ${ }^{a}, *$, Kuang-Yow Lian ${ }^{b}$, Cheung-Chieh Ku ${ }^{a}$, Che-Lun Su ${ }^{a}$ \\ a Department of Marine Engineering, National Taiwan Ocean University, Keelung, Taiwan, ROC \\ ${ }^{\mathrm{b}}$ Department of Electrical Engineering National Taipei University of Technology Taipei, Taiwan, ROC
}

\begin{abstract}
This document deals with the problem of passive fuzzy controller design with the state-derivative feedback approach for the nonlinear stochastic singular systems. Recently, the singular systems have a greater focus on literature because they can keep more physical system characteristics than conventional systems. At first, the Takagi-Sugeno fuzzy stochastic singular models are used to represent the nonlinear stochastic singular systems. Then, the state-derivative feedback approach and parallel distributed compensation method are employed to design the passive fuzzy controllers. In the design process, the Lyapunov stability conditions are developed subject to multiple performance constraints, including the passivity constraint and decay rate constraint. According to these Lyapunov stability conditions, the proposed fuzzy control problem can be effectively shifted into the linear matrix inequality problem that can be solved by using the convex optimal programming algorithm. At last, two examples are provided to verify the applicability and effectivity of the proposed passive fuzzy controller design approach.
\end{abstract}

Keywords: Takagi-Sugeno fuzzy stochastic singular systems, Fuzzy control with state-derivative feedback, Passivity constraint, Decay rate constraint

\section{Introduction}

$\mathrm{T}$ he singular systems [1-3] known as differential-algebraic systems, have drawn the attention of several researchers in recent years. Because the singular systems contain a particular case form in the state-space, unlike nonsingular systems, the singular systems can keep the system's physical characteristics more than the conventional systems. The singular systems are more complicated for stability analysis, and it is usually employed to describe a real system. Because of the front reason, the singular system has a significant effect on the field of research in control engineering. For theoretical and practical characteristics, the singular system has played an important role. In recent years, many researchers have spent a lot of effort on singular systems because the singular system will be stabilized once the only system is regularity and impulsefree [4-5]. Although many pieces of research have solved for the control problem in [6], the singular system still involves semi-defined and non-convex optimization terms, so it is challenging to solve numerically. It is well known that jumping behavior often occurs in electrical systems. Jumping behavior is a seemingly discontinuous change in the states of the system driven by constrained dynamics. However, jumping behavior may have singularities in the system. In 
[7], the authors proposed a method to solve these singularities for the electrical systems. This kind of singular system has many comfortable studies and applications in engineering, e.g., the power system [8] and the mechanical system [9].

In the past few decades, the Takagi-Sugeno (T-S) fuzzy modeling technique has become a popular and useful tool to represent a complex nonlinear control system [10-12]. By using the T-S fuzzy modeling method, the system dynamics of a nonlinear system can be constructed by mixing fuzzy sets based on linear local dynamic models. According to the T-S fuzzy models, several systems have been controlled by the T-S fuzzy control method [13]. When designing the fuzzy controller for corresponding T-S fuzzy models, one needs to get the mathematical model for the original nonlinear systems. Then, the overall complex nonlinear systems can be represented by "blending" of local linear sub-systems by way of membership functions. Based on the T-S fuzzy models, one can use various analysis approaches of linear systems to design the fuzzy controllers for nonlinear systems. Recently, the T-S fuzzy modeling method was also successfully applied to the nonlinear singular systems. The control problems for continuous-time and discrete-time T-S fuzzy singular models have been introduced and studied in [14-15]. In modern industrial systems, network control becomes more and more active. Considering the network control problem, the system, controller, sensor, and actuator are usually connected via a network. Due to the limitation of network bandwidth, network delays [16] and packet loss problems often exist in network systems [17]. In recent years, some scholars have studied the stability and performance of T-S fuzzy singular network control systems [18].

The stochastic behavior is usually seen as an unmeasured signal and is considered as a disturbance to the systems. By developing the stochastic system modeling approach, the system's unmeasured random signals were employed to construct a stochastic system. Random behavior usually affects the stability and performance of the system more than the perturbations. As the stability criteria for the stochastic systems are conservative, researchers must pay great attention to the analysis and synthesis of system stability. For stochastic systems, the passivity theory has been successfully applied to discuss stochastic behavior in the literature. In [19-20], the passivity theory was investigated to inhibit the disturbance of stochastic systems. The basic principle of passivity theory is developed in terms of system energy. It studies the energy changes from the input to the output of a system. Referring to [21-22], it can be found that a passive system is defined that the energy in the system is always dissipated or stored after some time. Using the passivity theory, one can analyze the physical system and design the controller through inputoutput relationships based on energy considerations. Some studies can be found in the literature to apply T-S fuzzy control techniques for nonlinear stochastic systems. In [23], the stability problem of stochastic singular systems has been investigated. Along with the criteria of stability, several performance indexes play critical issues in the control problems. The stability problem with other performance constraints for the stochastic singular systems has been investigated [24].

The state-derivative feedback method was well known as a useful method and can achieve the required performance for the control systems [25-26]. The motivation of using the state-derivative feedback rather than state feedback derives from some systems using accelerometers to measure the motion of the systems. Some applications of the state-derivative feedback method can be found in the literature, e.g., the prevention of vibration in mechanical systems [27], vibration control of bridge cables [28], control of wheel suspension systems [29], and vibration control of landing gear parts [30]. In the vibration suppression, it can be seen as a good performance for the controller maintaining steady-state stability of an uncontrolled system. We only can find some literature for the state-derivative feedback because it will cause the noise in the system to be amplified by differential amplification of the noise in the measured signal, and it is difficult to achieve perfect signal derivatives of signals in practice. According to the author's knowledge, there have fewer works that studied the control problem by using the state-derivative feedback method for the T-S fuzzy singular systems. In this paper, it is tried to develop a method to combine the state-derivative feedback method and passivity theory to simplify the passive fuzzy controller design approach with system requirements.

The purpose of this paper is to investigate a passive fuzzy control approach to satisfy the Lyapunov stability conditions and passivity requirements for nonlinear stochastic singular systems. Firstly, the nonlinear stochastic singular system is represented as a T-S fuzzy stochastic singular model. The corresponding T-S fuzzy controller can be designed by the Parallel Distributed Compensation (PDC) method [31-32]. Then, the overall fuzzy controller can be obtained by "blending" from the controllers of each rule based on the membership functions. 
The main characteristic of the suggested fuzzy control approach is to use the state-derivative feedback method to analyze the stability of the T-S fuzzy stochastic singular models. By employing the state-derivative feedback method, the complicated process of the passive fuzzy controller design can be reduced. However, only considering the stability is not enough for the designers; hence, the passivity constraint and decay rate are also considered in the suggested passive fuzzy control method. Several sufficient conditions are developed in this approach to satisfy the Lyapunov stability criteria, passivity constraint, and decay rate constraint. These sufficient conditions are effectively recast into the Linear Matrix Inequalities (LMI) form by Schur complement [33]. Then, the MATLAB LMI-toolbox can be used to solve the conditions to obtain feasible solutions. To verify the effectiveness and correctness of the proposed passive fuzzy control approach with state-derivative feedback, a numerical example is given to illustrate the control process for a nonlinear stochastic singular system.

The various parts of this paper are constructed as below. In Section II, a class of nonlinear stochastic singular systems is represented by a T-S fuzzy stochastic singular model. The control problem studied in this paper is also introduced in this section. In Section III, a complete introduction to the stability of the T-S fuzzy stochastic singular model, the state-derivative feedback method is employed to derive sufficient stability conditions in the disturbance-free situation. In Section IV, the passivity theory and state-derivative feedback method are used to design passive fuzzy controllers to deal with the external disturbances. The stability criteria, passivity constraint, and decay rate constraint are all considered in the sufficient conditions derivations. Using the LMI technique, the overall passive fuzzy controller can be obtained via solving these sufficient conditions. In Section V, the examples are given to show the applicability and effectivity of the suggested passive fuzzy control method. Finally, the conclusions are given in the last section.

\section{System descriptions and problem statements}

The problem of passive fuzzy controller design with state-derivative feedback is studied in this paper for the nonlinear stochastic singular systems. Considering the stochastic behaviors, the nonlinear stochastic singular systems can be expressed by the following T-S fuzzy stochastic singular model.
System Fuzzy Rule $i$ :

IF $\tilde{\rho}_{1}(t)$ is $M_{i 1}$ and $\ldots$ and $\tilde{\rho}_{n}(t)$ is $M_{i n}$ THEN

$\mathbf{S} \dot{x}(t)=\mathbf{A}_{i} x(t)+\mathbf{B}_{i} u(t)+\mathbf{G}_{i} v_{n}(t)$

$y(t)=\mathbf{C}_{i} x(t)+\mathbf{D}_{i} v_{n}(t)$

where $i=1,2, \cdots, r$ and $r$ is the fuzzy rules number, $\tilde{\rho}_{1}(t), \tilde{\rho}_{2}(t), \ldots, \tilde{\rho}_{n}(t)$ are the premise variables, $M_{i n}$ are fuzzy sets, $n$ is the number of premise variables, $x(t) \in \Re^{n}$ is the system state vector, $u(t) \in \Re^{m}$ is the control input vector, $y(t) \in \Re^{q}$ is the system output vector, $v_{n}(t) \in \Re^{j}$ is the external disturbance vector. $\mathbf{A}_{i} \in \Re^{n \times n}, \quad \mathbf{B}_{i} \in \Re^{n \times m}, \quad \mathbf{G}_{i} \in \Re^{n \times j}, \quad \mathbf{C}_{i} \in \Re^{q \times n}, \quad \mathbf{D}_{i} \in \Re^{q \times j}$ and $\mathbf{S} \in \Re^{n \times n}$ are constant matrices.

Let us consider the system state vector $x(t)$ and the control input vector $u(t)$ of the T-S fuzzy model (1), the T-S fuzzy model can be rewritten as follows:

$\mathbf{S} \dot{x}(t)=\sum_{i=1}^{r} \zeta_{i}(\tilde{\rho}(t))\left\{\mathbf{A}_{i} x(t)+\mathbf{B}_{i} u(t)+\mathbf{G}_{i} v_{n}(t)\right\}$

$y(t)=\sum_{i=1}^{r} \zeta_{i}(\tilde{\rho}(t))\left\{\mathbf{C}_{i} x(t)+\mathbf{D}_{i} v_{n}(t)\right\}$

where $\zeta_{i}(\tilde{\rho}(t))=\prod_{j=1}^{n} \mathbf{M}_{i j}\left(\tilde{\rho}_{j}(t)\right) / \sum_{i=1}^{r} \prod_{j=1}^{n} \mathbf{M}_{i j}\left(\tilde{\rho}_{j}(t)\right)$, $\zeta_{i}(\tilde{\rho}(t)) \geq 0, \quad \sum_{i=1}^{r} \zeta_{i}(\tilde{\rho}(t))=1$ and $\mathrm{M}_{i j}\left(\tilde{\rho}_{j}(t)\right)$ is the grade of the membership of $\tilde{\rho}_{j}(t)$ in $\mathrm{M}_{i j}$. The premise variables are $\tilde{\rho}(t)=\left[\begin{array}{llll}\tilde{\rho}_{1}(t) & \tilde{\rho}_{2}(t) & \ldots & \tilde{\rho}_{n}(t)\end{array}\right]$.

Via the PDC method, one can design the fuzzy controller for the T-S fuzzy model (1) by using the state-derivative feedback method. In the PDC method, each fuzzy control rule can be designed according to the same premise parts of the T-S fuzzy model. That is, the fuzzy controller has the same membership functions of the T-S fuzzy model. Thus, the overall state-derivative feedback fuzzy controller can be constructed by "blending" from those membership functions. The suggested state-derivative feedback fuzzy controller has the following form:

Fuzzy Controller Rule $i$ :

IF $\tilde{\rho}_{1}(t)$ is $M_{i 1}$ and $\ldots$ and $\tilde{\rho}_{n}(t)$ is $M_{i n}$ THEN

$u(t)=-\mathbf{F}_{i} \dot{x}(t)$

Then, the overall fuzzy controller can be represented as follows:

$u(t)=-\sum_{i=1}^{r} \zeta_{i}(\tilde{\rho}(t)) \mathbf{F}_{i} \dot{x}(t)$ 
Thus, the following closed-loop system can be got by substituting (4) into (2).

$$
\begin{aligned}
& \mathbf{S} \dot{x}(t)=\sum_{i=1}^{r} \sum_{j=1}^{r} \zeta_{i}(\tilde{\rho}(t)) \zeta_{j}(\tilde{\rho}(t))\left\{\mathbf{A}_{i} x(t)-\mathbf{B}_{i} \mathbf{F}_{j} \dot{x}(t)\right. \\
& \left.\quad+\mathbf{G}_{i} v_{n}(t)\right\} \\
& y(t)=\sum_{i=1}^{r} \zeta_{i}(\tilde{\rho}(t))\left\{\mathbf{C}_{i} x(t)+\mathbf{D}_{i} v_{n}(t)\right\}
\end{aligned}
$$

The first problem of the proposed fuzzy controller design is to find the constant gain matrices $\mathbf{F}_{j} \in \Re^{m \times n}$ such that the matrices $\left(\mathbf{S}+\mathbf{B}_{i} \mathbf{F}_{j}\right)$ to be full rank. It should be noted that the matrices $\left(\mathbf{S}+\mathbf{B}_{i} \mathbf{F}_{j}\right)$ have full rank if the following conditions hold.

$\operatorname{rank}\left[\mathbf{S}, \mathbf{B}_{i}\right]=n$

According to [34], it can be found that (6) is the necessary condition for the state-derivative feedback method applied in the controller design for singular systems. Some authors tried to apply the state feedback method and state-derivative feedback method to solve the controller design problem when the condition (6) does not hold. However, these methods lead to complex solving processes and unsuitable controller design. Therefore, it is assumed that the condition (6) is held in this paper.

It can be found that (5a) is equivalent to

$$
\begin{aligned}
& \left(\mathbf{S}+\sum_{k=1}^{r} \sum_{l=1}^{r} \zeta_{k}(\tilde{\rho}(t)) \zeta_{l}(\tilde{\rho}(t)) \mathbf{B}_{k} \mathbf{F}_{l}\right) \dot{x}(t)=\sum_{i=1}^{r} \zeta_{i}(\tilde{\rho}(t)) \\
& \quad \times\left\{\mathbf{A}_{i} x(t)+\mathbf{G}_{i} v_{n}(t)\right\}
\end{aligned}
$$

According to (6), one can obtain that $\left(\mathbf{S}+\mathbf{B}_{i} \mathbf{F}_{j}\right)$ is a full rank matrix. In this case, the inverse of $\left(\mathbf{S}+\mathbf{B}_{i} \mathbf{F}_{j}\right)$ exists as well as the matrix $\left(\mathbf{S}+\sum_{k=1}^{r} \sum_{l=1}^{r} \zeta_{k}(\tilde{\rho}(t)) \zeta_{l}(\tilde{\rho}(t)) \mathbf{B}_{k} \mathbf{F}_{l}\right)$ can be inversed. Therefore, (7) can be rewritten as follows:

$$
\begin{array}{r}
\dot{x}(t)=\left(\mathbf{S}+\sum_{k=1}^{r} \sum_{l=1}^{r} \zeta_{k}(\tilde{\rho}(t)) \zeta_{l}(\tilde{\rho}(t)) \mathbf{B}_{k} \mathbf{F}_{l}\right)^{-1} \sum_{i=1}^{r} \zeta_{i}(\tilde{\rho}(t)) \mathbf{A}_{i} x(t) \\
+\left(\mathbf{S}+\sum_{k=1}^{r} \sum_{l=1}^{r} \zeta_{k}(\tilde{\rho}(t)) \zeta_{l}(\tilde{\rho}(t)) \mathbf{B}_{k} \mathbf{F}_{l}\right)^{-1} \sum_{i=1}^{r} \zeta_{i}(\tilde{\rho}(t)) \mathbf{G}_{i} v_{n}(t)
\end{array}
$$

Remark 1

For any nonsymmetric matrix $\mathbf{Q} \in \Re^{n \times n}\left(\mathbf{Q} \neq \mathbf{Q}^{\mathrm{T}}\right)$, if $\mathbf{Q}+\mathbf{Q}^{\mathrm{T}}<0$ then $\mathbf{Q}$ is a full rank matrix.

The passivity theory developed in literature is a useful method to design controllers to achieve the energy requirements for the control systems. It is known that the characteristic of the passivity theory is that the attenuation performance can be achieved when the systems are affected by external disturbances. By using the definition of power supply functions, the passivity requirement is introduced below.

Definition 1. [19]

Given constant matrices $Z_{1}, Z_{2} \geq 0$ and $Z_{3}$. The system is called passive with the external disturbance $v_{n}(t)$ and system output $y(t)$ for all terminal time $t_{p}>0$ if the following inequality is satisfied.

$$
\begin{aligned}
& 2 \int_{0}^{t_{p}} y^{\mathrm{T}}(t) \mathbf{Z}_{1} v_{n}(t) d t>\int_{0}^{t_{p}} y^{\mathrm{T}}(t) \mathbf{Z}_{2} y(t) d t \\
& \quad+\int_{0}^{t_{p}} v_{n}^{\mathrm{T}}(t) \mathbf{Z}_{3} v_{n}(t) d t
\end{aligned}
$$

Note that inequality (9) can be used to define different performance requirements by setting different matrices $Z_{1}, Z_{2} \geq 0$, and $Z_{3}$. In this paper, let us consider the Strictly Input Passive Performance Constraint (SIPPC) by setting $\mathbf{Z}_{1} \triangleq I, \mathbf{Z}_{2} \triangleq \mathbf{0}$, $\mathbf{Z}_{3} \triangleq \theta \mathbf{I}$ and $\theta$ is a positive scalar. In this case, the passivity requirement (9) considered in this paper can be rewritten as

$2 \int_{0}^{t_{p}} y^{\mathrm{T}}(t) v_{n}(t) d t>\theta \int_{0}^{t_{p}} v_{n}^{T}(t) v_{n}(t) d t$

Considering the passivity constraint, the control problem of this paper is to find fuzzy control gains $\mathbf{F}_{j}$ for the state-derivative feedback passive fuzzy controller (3) such that the SIPPC (10) is satisfied. In order to solve this problem, the passivity theory and Lyapunov stability theory are employed to design the state-derivative feedback passive fuzzy controller (3).

\section{Stability condition derivations for Takagi- Sugeno fuzzy stochastic singular systems}

In order to study the stability analysis of T-S fuzzy singular systems, let us first discuss the derivations of stability conditions under the assumption of without considering external disturbances. Considering the T-S fuzzy system (5) with disturbance-free, the sufficient stability conditions for the closed-loop system can be obtained by the following theorem.

Theorem 1. The closed-loop T-S fuzzy system (5) with disturbance-free is asymptotically stable if 
there exists a positive definite matrix $\tilde{\mathbf{R}}$, feedback gains $\mathbf{K}_{l}$ satisfying the following conditions.

$$
\begin{gathered}
\mathbf{S} \tilde{\mathbf{R}} \mathbf{A}_{i}^{\mathrm{T}}+\mathbf{B}_{k} \mathbf{K}_{l} \mathbf{A}_{i}^{\mathrm{T}}+\mathbf{A}_{i} \tilde{\mathbf{R}} \mathbf{S}^{\mathrm{T}}+\mathbf{A}_{i} \mathbf{K}_{l}^{\mathrm{T}} \mathbf{B}_{k}^{\mathrm{T}}<0 \\
\quad \text { for } i, k, l=1 \ldots \ldots . r
\end{gathered}
$$

Proof:

Analyzing the stability of the closed-loop system (5), one can define the Lyapunov function as

$V(x(t))=x^{\mathrm{T}}(t) \mathbf{P} x(t)$

Taking differential of the Lyapunov function $V(x(t))$, one can get

$\dot{V}(x(t))=\dot{x}^{\mathrm{T}}(t) \mathbf{P} x(t)+x^{\mathrm{T}}(t) \mathbf{P} \dot{x}(t)$

In this theorem, the external disturbance is assumed to disappear. Under the assumption $v_{n}(t)=0$, equation (13) can be rewritten as follows by substituting (8) into (13).

$$
\begin{aligned}
& \dot{V}(x(t))=\left\{\left(\mathbf{S}+\sum_{k=1}^{r} \sum_{l=1}^{r} \zeta_{k}(\tilde{\rho}(t)) \zeta_{l}(\tilde{\rho}(t)) \mathbf{B}_{k} \mathbf{F}_{l}\right)^{-1}\right. \\
&\left.\sum_{i=1}^{r} \zeta_{i}(\tilde{\rho}(t)) \mathbf{A}_{i} x(t)\right\}^{\mathrm{T}} \mathbf{P} x(t) \\
&+x^{\mathrm{T}}(t) \mathbf{P}\left\{\left(\mathbf{S}+\sum_{k=1}^{r} \sum_{l=1}^{r} \zeta_{k}(\tilde{\rho}(t)) \zeta_{l}(\tilde{\rho}(t)) \mathbf{B}_{k} \mathbf{F}_{l}\right)^{-1}\right. \\
& \sum_{i=1}^{r} \zeta_{i}(\tilde{\rho}(t)) \mathbf{A}_{i} x(t)
\end{aligned}
$$

After arrangement (14) can be rewritten as

$$
\begin{aligned}
& \dot{V}(x(t))=x^{\mathrm{T}}(t)\left\{\sum_{i=1}^{r} \zeta_{i}(\tilde{\rho}(t)) \mathbf{A}_{i}^{\mathrm{T}}\right. \\
& \left.\qquad \mathbf{S}+\sum_{k=1}^{r} \sum_{l=1}^{r} \zeta_{k}(\tilde{\rho}(t)) \zeta_{l}(\tilde{\rho}(t)) \mathbf{B}_{k} \mathbf{F}_{l}\right)^{-\mathrm{T}} \mathbf{P} \\
& +\mathbf{P}\left(\mathbf{S}+\sum_{k=1}^{r} \sum_{l=1}^{r} \zeta_{k}(\tilde{\rho}(t)) \zeta_{l}(\tilde{\rho}(t)) \mathbf{B}_{k} \mathbf{F}_{l}\right)^{-1} \\
& \left.\sum_{i=1}^{r} \zeta_{i}(\tilde{\rho}(t)) \mathbf{A}_{i}\right\} x(t)
\end{aligned}
$$

By pre-multiplying

$\left(\mathbf{S}+\sum_{k=1}^{r} \sum_{l=1}^{r} \zeta_{k}(\tilde{\rho}(t)) \zeta_{l}(\tilde{\rho}(t)) \mathbf{B}_{k} \mathbf{F}_{l}\right) \mathbf{P}^{-1}$ and postmultiplying $\mathbf{P}^{-1}\left(\mathbf{S}+\sum_{k=1}^{r} \sum_{l=1}^{r} \zeta_{k}(\tilde{\rho}(t)) \zeta_{l}(\tilde{\rho}(t)) \mathbf{B}_{k} \mathbf{F}_{l}\right)^{\mathrm{T}}$ on both sides of (15), one can obtain

$$
\begin{gathered}
\dot{V}(x(t))=\sum_{i=1}^{r} \sum_{k=1}^{r} \sum_{l=1}^{r} \zeta_{i}(\tilde{\rho}(t)) \zeta_{k}(\tilde{\rho}(t)) \zeta_{l}(\tilde{\rho}(t)) \\
\times x^{\mathrm{T}}(t)\left[\left(\mathbf{S}+\mathbf{B}_{k} \mathbf{F}_{l}\right) \mathbf{P}^{-1} \mathbf{A}_{i}^{\mathrm{T}}+\mathbf{A}_{i} \mathbf{P}^{-1}\left(\mathbf{S}+\mathbf{B}_{k} \mathbf{F}_{l}\right)^{\mathrm{T}}\right] x(t) \\
=\sum_{i=1}^{r} \sum_{k=1}^{r} \sum_{l=1}^{r} \zeta_{i}(\tilde{\rho}(t)) \zeta_{k}(\tilde{\rho}(t)) \zeta_{l}(\tilde{\rho}(t)) \\
\times x^{\mathrm{T}}(t)\left[\mathbf{S P}^{-1} \mathbf{A}_{i}^{\mathrm{T}}+\mathbf{B}_{k} \mathbf{F}_{l} \mathbf{P}^{-1} \mathbf{A}_{i}^{\mathrm{T}}+\mathbf{A}_{i} \mathbf{P}^{-1} \mathbf{S}^{\mathrm{T}}+\mathbf{A}_{i} \mathbf{P}^{-1} \mathbf{F}_{l}^{\mathrm{T}} \mathbf{B}_{k}^{\mathrm{T}}\right] x(t)
\end{gathered}
$$

According to Remark 1, it is noted that the matrices $\left(\mathbf{S}+\mathbf{B}_{k} \mathbf{F}_{l}\right) \mathbf{P}^{-1} \mathbf{A}_{i}^{\mathrm{T}}$ are full rank, and so the matrices $\left(\mathbf{S}+\mathbf{B}_{k} \mathbf{F}_{l}\right)$ are also full rank.

Defining a variable $\tilde{\mathbf{R}}=\mathbf{P}^{-1}, \tilde{\mathbf{R}}>0$ and $\mathbf{K}_{l}=\mathbf{F}_{l} \tilde{\mathbf{R}}$, the equation (16) can be rewritten as follows:

$$
\begin{gathered}
\dot{V}(x(t))=\sum_{i=1}^{r} \sum_{k=1}^{r} \sum_{l=1}^{r} \zeta_{i}(\tilde{\rho}(t)) \zeta_{k}(\tilde{\rho}(t)) \zeta_{l}(\tilde{\rho}(t)) \\
\times x^{\mathrm{T}}(t)\left[\mathbf{S} \tilde{\mathbf{R}} \mathbf{A}_{i}^{\mathrm{T}}+\mathbf{B}_{k} \mathbf{K}_{l} \mathbf{A}_{i}^{\mathrm{T}}+\mathbf{A}_{i} \tilde{\mathbf{R}} \mathbf{S}^{\mathrm{T}}+\mathbf{A}_{i} \mathbf{K}_{l}^{\mathrm{T}} \mathbf{B}_{k}^{\mathrm{T}}\right] x(t)
\end{gathered}
$$

Thus, if the condition (11) is satisfied, then the closed-loop system (5) is asymptotically stable due to $\dot{V}(x(t))<0$.

The closed-loop singular system (5) with disturbance-free can be controlled by the state-derivative feedback fuzzy controller (3). Comparing with the traditional state feedback method, the proposed results developed by using the state-derivative feedback method are more convenient for the designers. However, the stability constraint is just the fundamental requirement for the control systems. To increase the contributions of the proposed statederivative feedback fuzzy controller design method, the SIPPC is considered when the T-S fuzzy singular system (5) considers the external disturbance. In addition to the SIPPC, the decay rate constraint is also considered in the next section.

\section{Passive fuzzy control with state-derivative feedback for Takagi-Sugeno fuzzy stochastic singular models}

In the previous section, the state-derivative feedback method was applied to derive the Lyapunov stability conditions for the T-S fuzzy singular systems with disturbance-free. Considering the external disturbances, the aim of this section is to derive stability conditions for the closed-loop system (5) such that the SIPPC described in (10) can be satisfied. Besides, the decay rate is also considered in the proposed passive fuzzy controller design process. Let us first apply the passivity theory to treat the external disturbances of systems. The sufficient stability conditions subject to SIPPC of (10) are developed in the following theorem. 
Theorem 2. The closed-loop system (5) is asymptotically stable and satisfies SIPPC of (10) if there exists a positive definite matrix $\tilde{\mathbf{R}}$, feedback gains $\mathbf{K}_{l}$ and matrices $Z_{1}, Z_{2} \geq 0$ and $Z_{3}$ satisfying the following sufficient conditions.

$$
\begin{gathered}
{\left[\begin{array}{c}
\mathbf{C}_{1}^{\mathrm{T}} \mathbf{Z}_{2} \mathbf{C}_{1}+\mathbf{S} \tilde{\mathbf{R}} \mathbf{A}_{i}^{\mathrm{T}}+\mathbf{B}_{k} \mathbf{K}_{l} \mathbf{A}_{i}^{\mathrm{T}}+\mathbf{A}_{i} \tilde{\mathbf{R}} \mathbf{S}^{\mathrm{T}}+\mathbf{A}_{i} \mathbf{K}_{l}^{\mathrm{T}} \mathbf{B}_{k}^{\mathrm{T}} \\
-\mathbf{Z}_{1}^{\mathrm{T}} \mathbf{C}_{1}+\mathbf{D}_{1}^{\mathrm{T}} \mathbf{Z}_{2} \mathbf{C}_{1}+\mathbf{G}_{i}^{\mathrm{T}} \\
* \\
\mathbf{Z}_{3}-\mathbf{D}_{1}^{\mathrm{T}} \mathbf{Z}_{1}-\mathbf{Z}_{1} \mathbf{D}_{1}+\mathbf{D}_{1}^{\mathrm{T}} \mathbf{Z}_{2} \mathbf{D}_{1}
\end{array}\right]<0}
\end{gathered}
$$

Proof:

Defining the same Lyapunov function, one has

$\dot{V}(x(t))=x^{\mathrm{T}}(t) \mathbf{P} x(t)$

Taking differential of the Lyapunov function $V(x(t))$, one can obtain
$\dot{V}(x(t))=\dot{x}^{\mathrm{T}}(t) \mathbf{P} x(t)+x^{\mathrm{T}}(t) \mathbf{P} \dot{x}(t)$

Substituting (8) into (20), $\dot{V}(x(t))$ can be rewritten as Equation (21) can be rewritten as follows after the appropriate arrangement.

Arranging the equation (22), one has

$\dot{V}(x(t))=\left[\begin{array}{l}x(t) \\ v_{n}(t)\end{array}\right]^{\mathrm{T}}\left[\begin{array}{ll}\Lambda & * \\ \Omega & 0\end{array}\right]\left[\begin{array}{l}x(t) \\ v_{n}(t)\end{array}\right]$

where

$$
\Lambda=\sum_{i=1}^{r} \zeta_{i}(\tilde{\rho}(t)) \mathbf{A}_{i}^{\mathrm{T}}\left(\mathbf{S}+\sum_{k=1}^{r} \sum_{l=1}^{r} \zeta_{k}(\tilde{\rho}(t)) \zeta_{l}(\tilde{\rho}(t)) \mathbf{B}_{k} \mathbf{F}_{l}\right)^{-\mathrm{T}} \mathbf{P}
$$$$
+\mathbf{P}\left(\mathbf{S}+\sum_{k=1}^{r} \sum_{l=1}^{r} \zeta_{k}(\tilde{\rho}(t)) \zeta_{l}(\tilde{\rho}(t)) \mathbf{B}_{k} \mathbf{F}_{l}\right)^{-1} \sum_{i=1}^{r} \zeta_{i}(\tilde{\rho}(t)) \mathbf{A}_{i} x(t)
$$

and

$$
\begin{aligned}
& \dot{V}(x(t))=\left\{\left(\mathbf{S}+\sum_{k=1}^{r} \sum_{l=1}^{r} \zeta_{k}(\tilde{\rho}(t)) \zeta_{l}(\tilde{\rho}(t)) \mathbf{B}_{k} \mathbf{F}_{l}\right)^{-1} \sum_{i=1}^{r} \zeta_{i}(\tilde{\rho}(t)) \mathbf{A}_{i} x(t)\right. \\
& \left.+\left(\mathbf{S}+\sum_{k=1}^{r} \sum_{l=1}^{r} \zeta_{k}(\tilde{\rho}(t)) \zeta_{l}(\tilde{\rho}(t)) \mathbf{B}_{k} \mathbf{F}_{l}\right)^{-1} \sum_{i=1}^{r} \zeta_{i}(\tilde{\rho}(t)) \mathbf{G}_{i} v_{n}(t)\right\} \mathbf{P} x(t) \\
& +x^{\mathrm{T}}(t) \mathbf{P}\left\{\left(\mathbf{S}+\sum_{k=1}^{r} \sum_{l=1}^{r} \zeta_{k}(\tilde{\rho}(t)) \zeta_{l}(\tilde{\rho}(t)) \mathbf{B}_{k} \mathbf{F}_{l}\right)^{-1} \sum_{i=1}^{r} \zeta_{i}(\tilde{\rho}(t)) \mathbf{A}_{i} x(t)\right. \\
& \left.+\left(\mathbf{S}+\sum_{k=1}^{r} \sum_{l=1}^{r} \zeta_{k}(\tilde{\rho}(t)) \zeta_{l}(\tilde{\rho}(t)) \mathbf{B}_{k} \mathbf{F}_{l}\right)^{-1} \sum_{i=1}^{r} \zeta_{i}(\tilde{\rho}(t)) \mathbf{G}_{i} v_{n}(t)\right\}
\end{aligned}
$$

$$
\begin{gathered}
\dot{V}(x(t))=x^{\mathrm{T}}(t)\left\{\sum_{i=1}^{r} \zeta_{i}(\tilde{\rho}(t)) \mathbf{A}_{i}^{\mathrm{T}}\left(\mathbf{S}+\sum_{k=1}^{r} \sum_{l=1}^{r} \zeta_{k}(\tilde{\rho}(t)) \zeta_{l}(\tilde{\rho}(t)) \mathbf{B}_{k} \mathbf{F}_{l}\right)^{-\mathrm{T}} \mathbf{P}\right. \\
\left.+\mathbf{P}\left(\mathbf{S}+\sum_{k=1}^{r} \sum_{l=1}^{r} \zeta_{k}(\tilde{\rho}(t)) \zeta_{l}(\tilde{\rho}(t)) \mathbf{B}_{k} \mathbf{F}_{l}\right)^{-1} \sum_{i=1}^{r} \zeta_{i}(\tilde{\rho}(t)) \mathbf{A}_{i}\right\} x(t) \\
+v_{n}^{\mathrm{T}}(t) \sum_{i=1}^{r} \zeta_{i}(\tilde{\rho}(t)) \mathbf{G}_{i}^{\mathrm{T}}\left(\mathbf{S}+\sum_{k=1}^{r} \sum_{l=1}^{r} \zeta_{k}(\tilde{\rho}(t)) \zeta_{l}(\tilde{\rho}(t)) \mathbf{B}_{k} \mathbf{F}_{l}\right)^{-\mathrm{T}} \mathbf{P} x(t) \\
+x^{\mathrm{T}}(t) \mathbf{P}\left(\mathbf{S}+\sum_{k=1}^{r} \sum_{l=1}^{r} \zeta_{k}(\tilde{\rho}(t)) \zeta_{l}(\tilde{\rho}(t)) \mathbf{B}_{k} \mathbf{F}_{l}\right)^{-1} \sum_{i=1}^{r} \zeta_{i}(\tilde{\rho}(t)) \mathbf{G}_{i} v_{n}(t)
\end{gathered}
$$


$\Omega=\sum_{i=1}^{r} \zeta_{i}(\tilde{\rho}(t)) \mathbf{G}_{i}^{\mathrm{T}}\left(\mathbf{S}+\sum_{k=1}^{r} \sum_{l=1}^{r} \zeta_{k}(\tilde{\rho}(t)) \zeta_{l}(\tilde{\rho}(t)) \mathbf{B}_{k} \mathbf{F}_{l}\right)^{-\mathrm{T}} \mathbf{P}$

Now, pre-multiplying

$\left[\begin{array}{ll}\left(\mathbf{S}+\sum_{k=1}^{r} \sum_{l=1}^{r} \zeta_{k}(\tilde{\rho}(t)) \zeta_{l}(\tilde{\rho}(t)) \mathbf{B}_{k} \mathbf{F}_{l}\right) \mathbf{P}^{-1} & 0 \\ 0 & \mathbf{I}\end{array}\right]$ and postmultiplying

$\left[\begin{array}{ll}\mathbf{P}^{-1}\left(\mathbf{S}+\sum_{k=1}^{r} \sum_{l=1}^{r} \zeta_{k}(\tilde{\rho}(t)) \zeta_{l}(\tilde{\rho}(t)) \mathbf{B}_{k} \mathbf{F}_{l}\right)^{\mathrm{T}} & \mathbf{0} \\ 0 & \mathbf{I}\end{array}\right]$ on both sides of (23), then one can obtain the following equation.

$\dot{V}(x(t))=\sum_{i=1}^{r} \sum_{k=1}^{r} \sum_{l=1}^{r} \boldsymbol{\zeta}_{i}(\tilde{\rho}(t)) \zeta_{k}(\tilde{\rho}(t)) \boldsymbol{\zeta}_{l}(\tilde{\rho}(t))\left[\begin{array}{l}x(t) \\ v_{n}(t)\end{array}\right]^{\mathrm{T}}$
$\times\left[\begin{array}{ll}\left(\mathbf{S}+\mathbf{B}_{k} \mathbf{F}_{l}\right) \mathbf{P}^{-1} \mathbf{A}_{i}^{\mathrm{T}}+\mathbf{A}_{i} \mathbf{P}^{-1}\left(\mathbf{S}+\mathbf{B}_{k} \mathbf{F}_{l}\right)^{\mathrm{T}} & * \\ \mathbf{G}_{i}^{\mathrm{T}} & 0\end{array}\right]\left[\begin{array}{l}x(t) \\ v_{n}(t)\end{array}\right]$

Let $\tilde{\mathbf{R}}=\mathbf{P}^{-1}, \tilde{\mathbf{R}}>0$ and $\mathbf{K}_{l}=\mathbf{F}_{l} \tilde{\mathbf{R}}$, then equation (24) can be rewritten as

$$
\begin{aligned}
& \dot{V}(x(t))=\sum_{i=1}^{r} \sum_{k=1}^{r} \\
& \quad \times \sum_{l=1}^{r} \zeta_{i}(\tilde{\rho}(t)) \zeta_{k}(\tilde{\rho}(t)) \zeta_{l}(\tilde{\rho}(t))\left[\begin{array}{l}
x(t) \\
v_{n}(t)
\end{array}\right]^{\mathrm{T}}\left[\begin{array}{ll}
\Theta & * \\
\mathrm{G}_{i}^{\mathrm{T}} & 0
\end{array}\right] \\
& \quad \times\left[\begin{array}{l}
x(t) \\
v_{n}(t)
\end{array}\right]
\end{aligned}
$$

where $\Theta=\mathbf{S} \tilde{\mathbf{R}} \mathbf{A}_{i}^{\mathrm{T}}+\mathbf{B}_{k} \mathbf{K}_{l} \mathbf{A}_{i}^{\mathrm{T}}+\mathbf{A}_{i} \tilde{\mathbf{R}} \mathbf{S}^{\mathrm{T}}+\mathbf{A}_{i} \mathbf{K}_{l}^{\mathrm{T}} \mathbf{B}_{k}^{\mathrm{T}}$.

Let us define a cost function as

$$
\begin{aligned}
\Xi\left(x(t), v_{n}(t)\right)= & \int_{0}^{t_{p}} y^{\mathrm{T}}(t) \mathbf{Z}_{2} y(t)+v_{n}^{\mathrm{T}}(t) \mathbf{Z}_{3} v_{n}(t) \\
& -2 y^{\mathrm{T}}(t) \mathbf{Z}_{1} v_{n}(t) d t \\
= & \int_{0}^{t_{p}} y^{\mathrm{T}}(t) \mathbf{Z}_{2} y(t)+v_{n}^{\mathrm{T}}(t) \mathbf{Z}_{3} v_{n}(t) \\
& -2 y^{\mathrm{T}}(t) \mathbf{Z}_{1} v_{n}(t)+\dot{V}(x(t))-V\left(x\left(t_{p}\right)\right) \\
\leq & \int_{0}^{t_{p}} \Psi\left(x(t), v_{n}(t)\right) d t
\end{aligned}
$$

where

$\Psi\left(x(t), v_{n}(t)\right)=y^{\mathrm{T}}(t) \mathbf{Z}_{2} y(t)+v_{n}^{\mathrm{T}}(t) \mathbf{Z}_{3} v_{n}(t)-2 y^{\mathrm{T}}(t) \mathbf{Z}_{1} v_{n}(t)$ $+\dot{V}(x(t))$

Substituting (2b) and (25) into (27) yields

$$
\begin{aligned}
& \Psi\left(x(t), v_{n}(t)\right)=\sum_{i=1}^{r} \sum_{k=1}^{r} \\
& \quad \times \sum_{l=1}^{r} \zeta_{i}(\tilde{\rho}(t)) \zeta_{k}(\tilde{\rho}(t)) \zeta_{l}(\tilde{\rho}(t))\left[\begin{array}{l}
x(t) \\
v_{n}(t)
\end{array}\right]^{\mathrm{T}} \Lambda\left[\begin{array}{l}
x(t) \\
v_{n}(t)
\end{array}\right]
\end{aligned}
$$

where $\Lambda=$

$\left[\begin{array}{c}\mathbf{C}_{1}^{\mathrm{T}} \mathbf{Z}_{2} \mathbf{C}_{1}+\Theta \\ -\mathbf{Z}_{1}^{\mathrm{T}} \mathbf{C}_{1}+\mathbf{D}_{1}^{\mathrm{T}} \mathbf{Z}_{2} \mathbf{C}_{1}+\mathbf{G}_{i}^{\mathrm{T}} \mathbf{Z}_{3}-\mathbf{D}_{1}^{\mathrm{T}} \mathbf{Z}_{1}-\mathbf{Z}_{1} \mathbf{D}_{1}+\mathbf{D}_{1}^{\mathrm{T}} \mathbf{Z}_{2} \mathbf{D}_{1}\end{array}\right]$

The satisfaction of condition (18) leads to $\Lambda<0$ that implies $\Psi\left(x(t), v_{n}(t)\right)<0$. From (26), one can find that $\Psi\left(x(t), v_{n}(t)\right)<0$ implies

$\Xi\left(x(t), v_{n}(t)\right)<0$

or

$$
\begin{aligned}
& 2 \int_{0}^{t_{p}} y^{\mathrm{T}}(t) \mathbf{Z}_{1} v_{n}(t) d t>\int_{0}^{t_{p}} y^{\mathrm{T}}(t) \mathbf{Z}_{2} y(t) d t \\
& \quad+\int_{0}^{t_{p}} v_{n}^{\mathrm{T}}(t) \mathbf{Z}_{3} v_{n}(t) d t
\end{aligned}
$$

Since (30) is equivalent to (9), it can be concluded that the closed-loop system (5) satisfies the passivity constraint. Subsequently, it is necessary to prove the stability of the closed-loop system. By assuming $v_{n}(t)=0$, the following inequality can be obtained from (28) with the condition (18).

$$
\begin{aligned}
& \dot{V}(x(t)) \leq \sum_{i=1}^{r} \sum_{k=1}^{r} \sum_{l=1}^{r} \zeta_{i}(\tilde{\rho}(t)) \zeta_{k}(\tilde{\rho}(t)) \zeta_{l}(\tilde{\rho}(t)) x^{\mathrm{T}}(t) \\
& \left(\mathbf{C}_{1}^{\mathrm{T}} \mathbf{Z}_{2} \mathbf{C}_{1}+\Theta\right) x(t)
\end{aligned}
$$

Obviously, if $\mathrm{Z}_{2} \geq 0$ is held, then $\dot{V}(x(t)) \leq 0$ can be easily found from condition (31). Since $\dot{V}(x(t)) \leq 0$, the closed-loop system (5) is therefore asymptotically stable.

From Theorem 2, it has been shown that the statederivative feedback passive fuzzy controller (3) can be employed to control the T-S fuzzy stochastic singular system (5) such that the closed-loop system is asymptotically stable and satisfies passivity constraint described in Definition 1. The conditions (18) derived in Theorem 2 are of LMI forms that can 
be solved by the convex optimization algorithm and MATLAB LMI-Toolbox. The passivity constraint led to better steady-state performance for the controlled systems. Another aspect, the decay rate constraint will bring better transient performance for the closed-loop systems. Hence, the passivity requirement is combined with the decay rate constraint in the subsequent passive fuzzy controller design process. For the T-S fuzzy stochastic singular system (5), the passive state-derivative feedback fuzzy controller design with the decay rate can be accomplished in the following theorem.

Theorem 3. The closed-loop system (5) is asymptotically stable and satisfies SIPPC of (10) as well as decay rate constraint if there exist positive definite matrices $\tilde{\mathbf{R}}, \mathbf{K}_{l}$, decay rate $\gamma$ and matrices $\mathbf{Z}_{1}, \mathbf{Z}_{2} \geq \mathbf{0}$ and $Z_{3}$ satisfying the following stability conditions.

$$
\begin{aligned}
& {\left[\begin{array}{l}
\mathbf{C}_{1}^{\mathrm{T}} \mathbf{Z}_{2} \mathbf{C}_{1}+\Theta+\gamma \mathbf{P} \quad * \\
-\mathbf{Z}_{1}^{\mathrm{T}} \mathbf{C}_{1}+\mathbf{D}_{1}^{\mathrm{T}} \mathbf{Z}_{2} \mathbf{C}_{1}+\mathbf{G}_{i}^{\mathrm{T}} \quad \mathbf{Z}_{3}-\mathbf{D}_{1}^{\mathrm{T}} \mathbf{Z}_{1}-\mathbf{Z}_{1} \mathbf{D}_{1}+\mathbf{D}_{1}^{\mathrm{T}} \mathbf{Z}_{2} \mathbf{D}_{1}
\end{array}\right]} \\
& \quad<0 \text { for } i, k, l=1,2, \ldots, r
\end{aligned}
$$

where $\Theta=\mathbf{S} \tilde{\mathbf{R}} \mathbf{A}_{i}^{\mathrm{T}}+\mathbf{B}_{k} \mathbf{K}_{l} \mathbf{A}_{i}^{\mathrm{T}}+\mathbf{A}_{i} \tilde{\mathbf{R}} \mathbf{S}^{\mathrm{T}}+\mathbf{A}_{i} \mathbf{K}_{l}^{\mathrm{T}} \mathbf{B}_{k}^{\mathrm{T}}$.

Proof:

The condition (32) can be represented as follows:

$$
\begin{aligned}
& {\left[\begin{array}{ll}
\mathbf{C}_{1}^{\mathrm{T}} \mathrm{S}_{2} \mathbf{C}_{1}+\Theta & * \\
-\mathbf{Z}_{1}^{\mathrm{T}} \mathbf{C}_{1}+\mathbf{D}_{1}^{\mathrm{T}} \mathbf{Z}_{2} \mathbf{C}_{1}+\mathbf{G}_{i}^{\mathrm{T}} \mathbf{Z}_{3}-\mathbf{D}_{1}^{\mathrm{T}} \mathbf{Z}_{1}-\mathbf{Z}_{1} \mathbf{D}_{1}+\mathbf{D}_{1}^{\mathrm{T}} \mathbf{Z}_{2} \mathbf{D}_{1}
\end{array}\right]} \\
& +\left[\begin{array}{lr}
\gamma \mathbf{P} & 0 \\
0 & 0
\end{array}\right]<0
\end{aligned}
$$

Furthermore, the inequation (33) can be rewritten as follows:

$\tilde{\Gamma}<\left[\begin{array}{ll}-\gamma \mathbf{P} & 0 \\ 0 & 0\end{array}\right]$

where

$\tilde{\Gamma}=\left[\begin{array}{l}\mathbf{C}_{1}^{\mathrm{T}} \mathbf{Z}_{2} \mathbf{C}_{1}+\Theta \\ -\mathbf{Z}_{1}^{\mathrm{T}} \mathbf{C}_{1}+\mathbf{D}_{1}^{\mathrm{T}} \mathbf{Z}_{2} \mathbf{C}_{1}+\mathbf{G}_{i}^{\mathrm{T}} \mathbf{Z}_{3}-\mathbf{D}_{1}^{\mathrm{T}} \mathbf{Z}_{1}-\mathbf{Z}_{1} \mathbf{D}_{1}+\mathbf{D}_{1}^{\mathrm{T}} \mathbf{Z}_{2} \mathbf{D}_{1}\end{array}\right]$

The inequality (34) indicates that the condition (32) satisfies the decay rate performance for $\gamma>0$. From (34), it can be found that $\tilde{\Gamma}<0$ is held because $\gamma>0$ and $\mathbf{P}>0$. Note that $\tilde{\Gamma}<0$ implies the conditions (18) of Theorem 2 are satisfied. From Theorem 2 , it can be found that if the conditions of (18) are satisfied, then the closed-loop system (5) is

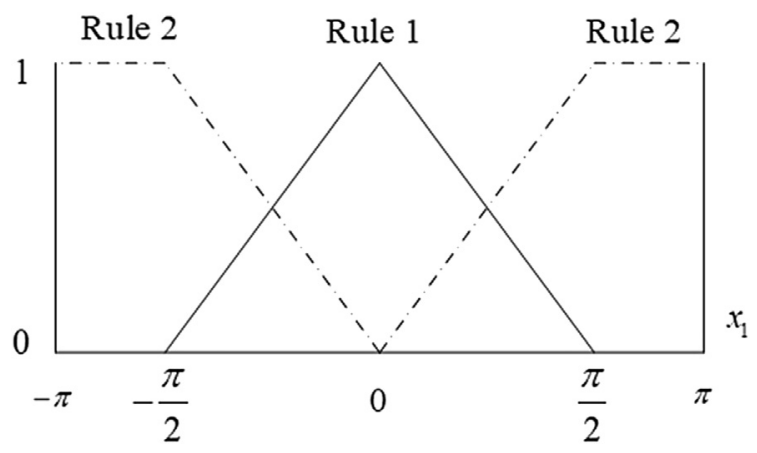

Fig. 1. Membership functions of $x_{1}(t)$ for Example 1.

asymptotically stable and satisfied SIPPC of (10). Therefore, the T-S fuzzy stochastic singular system (5) controlled by the state-derivative feedback fuzzy controller achieves SIPPC of (10), asymptotical stability, and decay rate constraint if conditions of (32) are satisfied.

The feasible solutions to the conditions of Theorem 3 can be solved via the LMI technique by the MATLAB LMI-Toolbox. Then, the passive fuzzy controller can be designed by the state-derivative feedback control method subject to SIPPC and decay rate constraints. In the next section, a numerical example is given to verify the applicability and effectiveness of the proposed passive fuzzy controller design approach.

\section{Simulation examples}

In this section, some examples are presented to verify the practicality and applicability of the proposed fuzzy controller design method.

Example 1. Consider a DC motor system described in [35]. The dynamic equations for the motions of the system are given as follows:

$\dot{x}_{1}(t)=x_{2}(t)$

$\dot{x}_{2}(t)=\frac{g}{l} \sin x_{1}(t)+\frac{N K_{m}}{m l^{2}} x_{3}$

$L_{a} \dot{x}_{3}(t)=K_{b} N x_{2}(t)-R\left(\eta_{t}\right) x_{3}(t)+u(t)+0.1 v_{n}(t)$

$y(t)=x_{1}(t)+v_{n}(t)$

where $x_{1}(t)=\theta_{p}(t), x_{2}(t)=\dot{\theta}_{p}(t), x_{3}(t)=I_{a}(t), u(t)$ is the control input and $v_{n}(t)$ is the external disturbance. $K_{m}$ is the motor torque constant, $K_{b}$ is the back emf constant, $N$ is the gear ratio. The parameters are set as follows: $L_{a}=\epsilon H$ with $\epsilon=0, g=$ $9.8 \mathrm{~m} / \mathrm{s}^{2}, l=1 \mathrm{~m}, m=1 \mathrm{~kg}, N=10, K_{m}=0.1 \mathrm{Nm} / A$, $K_{b}=0.1 \mathrm{Vs} / \mathrm{rad}, R\left(\eta_{t}\right)=1 \Omega$. 
The SIPPC of (10) is used to design passive fuzzy controller to achieve the attenuation performance. Here, let us set the parameter $\theta=1$. Let us consider the above nonlinear system, which can be transferred into a two-rule T-S fuzzy model with membership functions of Fig. 1 as follows:

Rule 1: IF $x_{1}(t)$ is about 0 THEN

$\mathbf{S} \dot{x}(t)=\mathbf{A}_{1} x(t)+\mathbf{B}_{1} u(t)+\mathbf{G}_{1} v_{n}(t)$

$y(t)=\mathbf{C}_{1} x(t)+\mathbf{D}_{1} v_{n}(t)$

Rule 2: IF $x_{1}(t)$ is about $\pm \frac{\pi}{2}$ THEN

$\mathbf{S} \dot{x}(t)=\mathbf{A}_{2} x(t)+\mathbf{B}_{2} u(t)+\mathbf{G}_{2} v_{n}(t)$

$y(t)=\mathbf{C}_{2} x(t)+\mathbf{D}_{2} v_{n}(t)$

The system parameters are given as follows:

$$
\begin{aligned}
\mathbf{S} & =\left[\begin{array}{lll}
1 & 0 & 0 \\
0 & 1 & 0 \\
0 & 0 & 0
\end{array}\right], \mathbf{A}_{1}=\left[\begin{array}{lll}
0 & 1 & 0 \\
9.8 & 0 & 1 \\
0 & 1 & -1
\end{array}\right], \\
\mathbf{A}_{2} & =\left[\begin{array}{lll}
0 & 1 & 0 \\
6.2389 & 0 & 1 \\
0 & 1 & -1
\end{array}\right], \\
\mathbf{B}_{1}=\mathbf{B}_{2} & =\left[\begin{array}{l}
0 \\
0 \\
1
\end{array}\right], \mathbf{C}_{1}=\mathbf{C}_{2}=\left[\begin{array}{lll}
1 & 0 & 0
\end{array}\right], \mathbf{G}_{1}=\mathbf{G}_{2} \\
& =\left[\begin{array}{l}
0 \\
0 \\
1
\end{array}\right], \mathbf{D}_{1}=\mathbf{D}_{2}=1
\end{aligned}
$$

By using LMI-Toolbox of MATLAB to solve the conditions of Theorem 2, the common positive

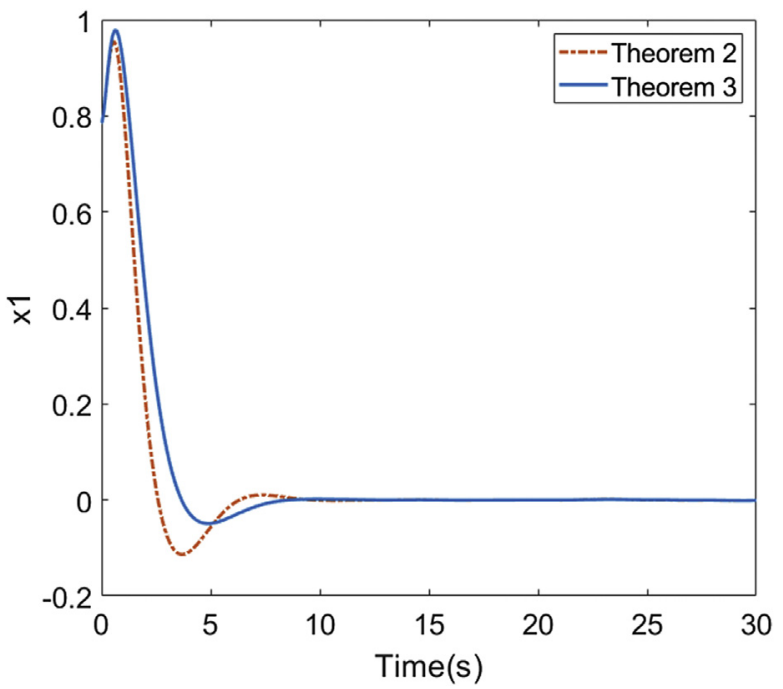

Fig. 2. Response comparisons of state $x_{1}(t)$ for Example 1 .

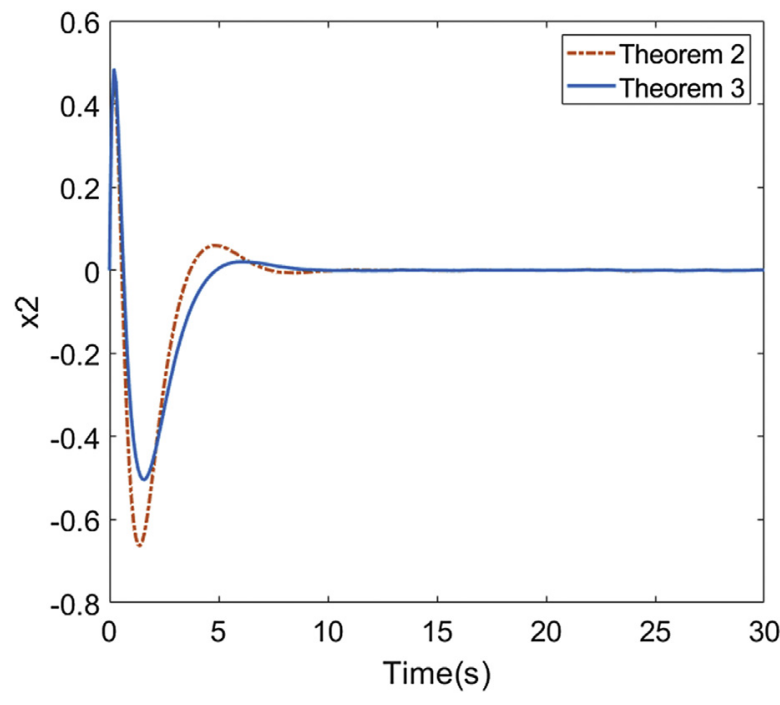

Fig. 3. Response comparisons of state $x_{2}(t)$ for Example 1.

definite matrix $\mathbf{P}$ and feedback gains of the fuzzy controller can be obtained as follows:

$\mathbf{P}=\left[\begin{array}{lll}0.2902 & 0.0954 & 0.0185 \\ 0.0954 & 0.0392 & 0.0056 \\ 0.0185 & 0.0056 & 0.0014\end{array}\right]$

$F_{1}=\left[\begin{array}{lll}-26.0122 & -10.9193 & -1.4937\end{array}\right]$

$F_{2}=\left[\begin{array}{lll}-26.0122 & -10.9193 & -1.4937\end{array}\right]$

According to the above feedback gains, the statederivative feedback fuzzy controller can be constructed by using the PDC approach as follows: Rule 1: IF $x_{1}(t)$ is about 0 THEN

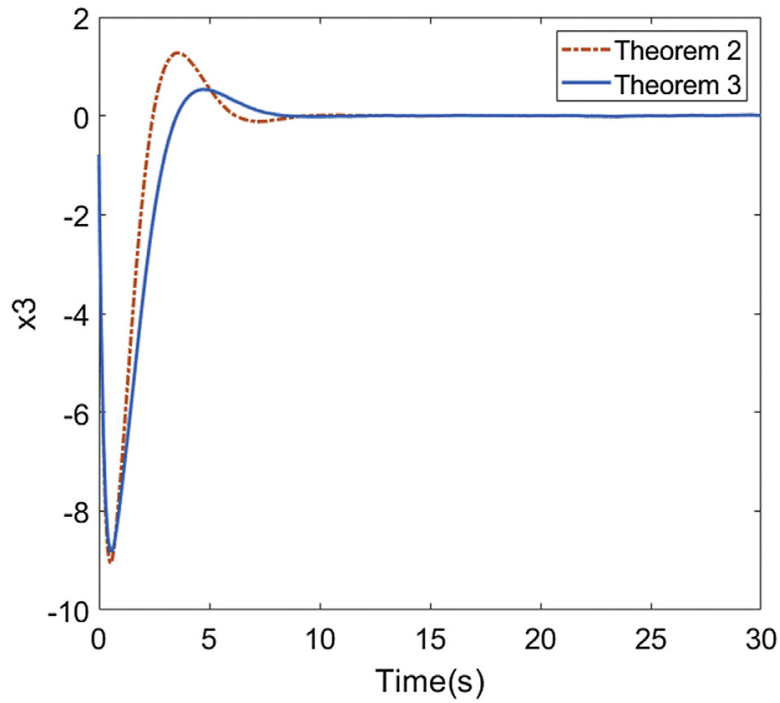

Fig. 4. Response comparisons of state $x_{3}(t)$ for Example 1. 
$u(t)=-\mathbf{F}_{1} \dot{x}(t)$

Rule 2: IF $x_{1}(t)$ is about $\pm \frac{\pi}{2}$ THEN

$u(t)=-\mathrm{F}_{2} \dot{x}(t)$

Considering the decay rate and solving the conditions of Theorem 3 via MATLAB LMI-Toolbox, one can get feasible solutions as follows:

$\mathbf{P}=\left[\begin{array}{lll}0.3707 & 0.1288 & 0.0239 \\ 0.1288 & 0.0536 & 0.0077 \\ 0.0239 & 0.0077 & 0.0018\end{array}\right]$

$\mathbf{F}_{1}=\left[\begin{array}{lll}-34.9940 & -14.8215 & -1.9833\end{array}\right]$

$F_{2}=\left[\begin{array}{lll}-34.9940 & -14.8215 & -1.9833\end{array}\right]$

For the simulations, the initial condition is chosen as $x(0)=\left[\begin{array}{lll}\frac{\pi}{4} & 0 & -\frac{\pi}{4}\end{array}\right]^{\mathrm{T}}$. From the responses of simulated results, the following specific value can be calculated to verify the SIPPC of (10).

For Theorem 2: $\frac{2 \int_{0}^{t_{p}} y^{\mathrm{T}}(t) v(t) d t}{\theta \int_{0}^{t_{p}} v^{T}(t) v(t) d t}=2.0732$

For Theorem 3: $\frac{2 \int_{0}^{t_{p}} y^{\mathrm{T}}(t) v(t) d t}{\theta \int_{0}^{t_{p}} v^{T}(t) v(t) d t}=2.0672$

The simulation responses of system states were shown in Fig. 2, Fig. 3 and Fig. 4. These figures showed the comparisons between simulation responses of Theorem 2 and Theorem 3. From these figures, one can find that Theorem 3 provided more rapid responses than Theorem 2 because the decay rate was considered in Theorem 3.

Example 2. In this example, the proposed passive fuzzy controller design method was compared with the fuzzy control approach developed in [36].

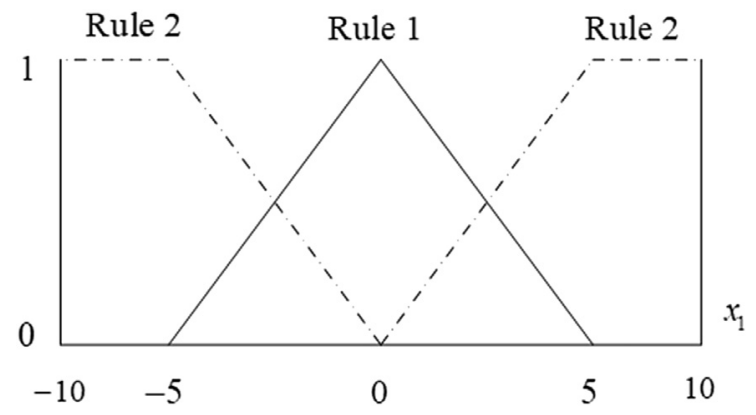

Fig. 5. Membership functions of $x_{1}(t)$ for Example 2.
Consider the following T-S fuzzy singular system described in [36].

Rule 1: IF $x_{1}(t)$ is about 0 THEN

$\mathbf{S} \dot{x}(t)=\mathbf{A}_{1} x(t)+\mathbf{B}_{1} u(t)+\mathbf{G}_{1} v_{n}(t)$

$y(t)=\mathbf{C}_{1} x(t)+\mathbf{D}_{1} v_{n}(t)$

Rule 2: IF $x_{1}(t)$ is about \pm 5 THEN

$\mathbf{S} \dot{x}(t)=\mathbf{A}_{2} x(t)+\mathbf{B}_{2} u(t)+\mathbf{G}_{2} v_{n}(t)$

$y(t)=\mathrm{C}_{2} x(t)+\mathrm{D}_{2} v_{n}(t)$

The system parameters are given as follows:

$$
\begin{aligned}
& \mathbf{S}=\left[\begin{array}{lll}
1 & 1 & 0 \\
2 & -5 & 0 \\
-2 & 3.5 & 0
\end{array}\right], \mathbf{A}_{1}=\left[\begin{array}{lll}
-4 & -1 & 0 \\
5 & 8 & 1 \\
1 & 5 & 1
\end{array}\right] \\
& \mathbf{A}_{2}=\left[\begin{array}{lll}
-4 & -1 & 1 \\
-4 & 8 & -3 \\
1 & 5 & 1
\end{array}\right]
\end{aligned}
$$

$$
\begin{gathered}
\mathbf{B}_{1}=\left[\begin{array}{l}
1 \\
0.2 \\
1
\end{array}\right], \mathbf{B}_{2}=\left[\begin{array}{l}
1 \\
0.6 \\
2
\end{array}\right], \mathbf{C}_{1}=\mathbf{C}_{2}=\left[\begin{array}{lll}
0 & 1 & 0
\end{array}\right], \mathbf{G}_{1} \\
=\mathbf{G}_{2}=\left[\begin{array}{l}
0.1 \\
0 \\
0
\end{array}\right], \mathbf{D}_{1}=\mathbf{D}_{2}=1
\end{gathered}
$$

Consider the membership functions of $x_{1}(t)$ shown in Fig. 5. By using the LMI-Toolbox of MATLAB, one can solve the conditions of Theorem 3 with the decay rate constraint. The passivity constraint parameter was set as $\theta=1$. Then, the common positive definite matrix $\mathbf{P}$ and state-

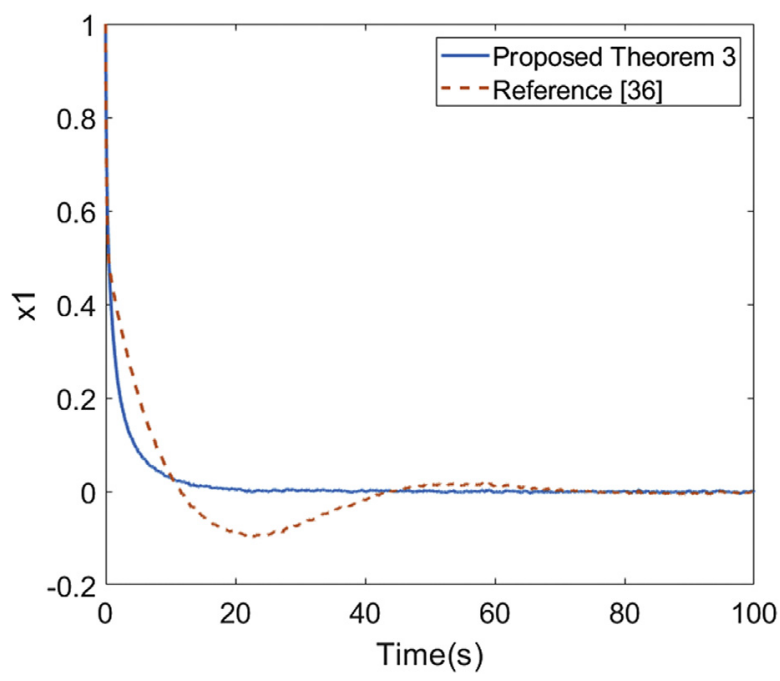

Fig. 6. Response comparisons of state $x_{1}(t)$ for Example 2 . 


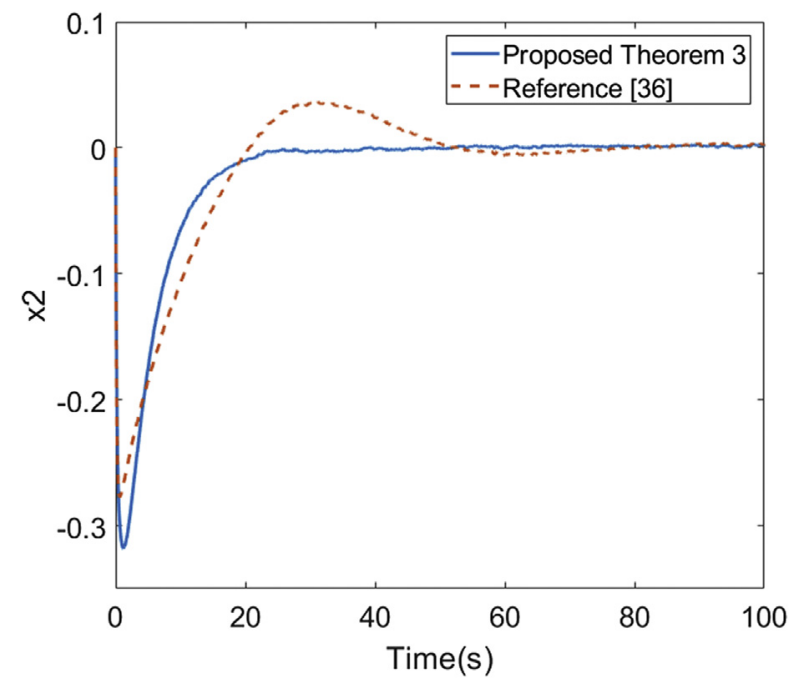

Fig. 7. Response comparisons of state $x_{2}(t)$ for Example 2.

derivative feedback gains of the proposed fuzzy controller can be obtained as follows:

$$
\begin{aligned}
& \mathbf{P}=\left[\begin{array}{lll}
0.2902 & 0.0954 & 0.0185 \\
0.0954 & 0.0392 & 0.0056 \\
0.0185 & 0.0056 & 0.0014
\end{array}\right] \\
& \mathbf{F}_{1}=\left[\begin{array}{lll}
2.9202 & -4.5618 & -0.3619
\end{array}\right] \\
& \mathbf{F}_{2}=\left[\begin{array}{lll}
2.9202 & -4.5618 & -0.3619
\end{array}\right]
\end{aligned}
$$

According to the above feedback gains, the statederivative feedback fuzzy controller can be constructed by using the PDC approach as follows:

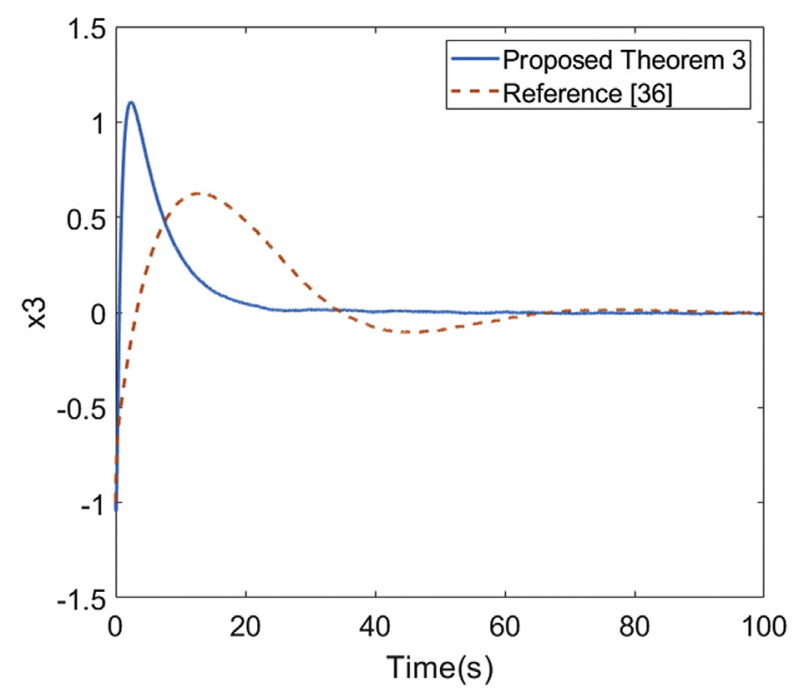

Fig. 8. Response comparisons of state $x_{3}(t)$ for Example 2.
Rule 1: IF $x_{1}(t)$ is about 0 THEN

$u(t)=-\mathrm{F}_{1} \dot{x}(t)$

Rule 2: IF $x_{1}(t)$ is about \pm 5 THEN

$u(t)=-\mathbf{F}_{2} \dot{x}(t)$

On the other hand, the feedback gains solved by the design method of [36] can be obtained as follows:

$\mathbf{F}_{1}=\left[\begin{array}{lll}-8.5415 & 2.7644 & -9.0762\end{array}\right]$

$\mathbf{F}_{2}=\left[\begin{array}{lll}-7.5421 & 6.1982 & -8.4471\end{array}\right]$

The initial condition of this example is chosen as $x(0)=\left[\begin{array}{lll}1 & 0 & -1\end{array}\right]^{\mathrm{T}}$. From the responses of simulated results, the following specific value can be calculated to verify the SIPPC of (10). In addition, the simulation responses of the states are shown in Fig. 6, Fig. 7 and Fig. 8.

Theorem 3: $\frac{{ }^{2} \int_{0}^{t_{p}} y^{\mathrm{T}}(t) v(t) d t}{{ }_{\theta}^{t_{p}} \int_{0}^{T}(t) v(t) d t}=2.0142$

It can be found that the values of (43a), (43b), and (49) are all bigger than 1. It implies the closed-loop system achieving the SIPPC via the proposed passive fuzzy controller. The comparisons of settling time between the proposed passive fuzzy control method and the design method of [36] were given in Table 1. From Table 1, one can find that the proposed passive fuzzy control method has a shorter settling time. Besides, the comparisons of state variances were given in Table 2. From Table 2, one can find that the proposed design method also has a better ability to inhibit disturbance than the approach of [36]. It can be concluded that a better transient and steady-state response can be obtained by using the passive fuzzy controller that was designed via solving the conditions of Theo-

Table 1. Comparisons of settling time.

\begin{tabular}{lll}
\hline Settling Time & Theorem 3 & Reference [36] \\
\hline$x_{1}(t)$ & 25 (sec.) & 70 (sec.) \\
$x_{2}(t)$ & 40 (sec.) & $85(\mathrm{sec})$. \\
$x_{3}(t)$ & 40 (sec.) & $95(\mathrm{sec})$. \\
\hline
\end{tabular}

Table 2. Comparisons of system state variances.

\begin{tabular}{lll}
\hline State Variance & Theorem 3 & Reference [36] \\
\hline$x_{1}(t)$ & 0.0098 & 0.0151 \\
$x_{2}(t)$ & 0.0037 & 0.0041 \\
$x_{3}(t)$ & 0.0590 & 0.0635 \\
\hline
\end{tabular}


rem 3. Applying the proposed passive fuzzy controller design method, the nonlinear stochastic singular systems can be controlled to satisfy stability, passivity, and decay rate constraints simultaneously.

\section{Conclusions}

In this paper, a methodology of passive fuzzy controller design has been studied for fulfilling multiple performance requirements for continuoustime nonlinear stochastic singular systems that were represented by the T-S fuzzy stochastic singular models. The performance requirements described in this approach included the system stability, the decay rate constraint, and the passivity requirement. Sufficient conditions have been derived to meet the previously mentioned multiple performance requirements. Employing the LMI method to solve these sufficient conditions, the state-derivative feedback approach has been used to design a passive fuzzy controller for the T-S fuzzy stochastic singular models. In the end, the practicality and applicability of the proposed passive fuzzy control method have been verified by the examples. Obviously, it can be seen that better transient behavior, such as settling time and rising time, can be obtained by considering the decay rate constraint. Via the passivity theory, better steady-state performance can be obtained after effectively attenuating the external disturbances.

\section{Acknowledgments}

The authors would like to express their sincere gratitude to the anonymous reviewers who gave us many constructive comments and suggestions. This work was supported by the Ministry of Science and Technology of the Republic of China under Contract MOST108-2221-E-019-061. It was also supported by the Joint Research Program Funding Sponsorship by University System of Taipei under Grant Number USTP-NTUTNTOU-109-03.

\section{References}

[1] Ligang W, Shi P, Gao H. State estimation and sliding-mode control of Markovian jump singular systems. IEEE Trans Automat Contr 2010;55(No. 5):1213-9.

[2] Peng S, Wang H, Lim CC. Network-based event-triggered control for singular systems with quantizations. IEEE Trans Ind Elect 2015;63(No. 2):1230-8.

[3] Yao D, Ren H, Li P, Zhou Q. Sliding mode output-feedback control of discrete-time Markov jump systems using singular system method. J Franklin Inst 2018;355(No.13): 5576-91.

[4] Lu G, Ho DWC. Generalized quadratic stability for continuous-time singular systems with nonlinear perturbation. IEEE Trans Automat Contr 2006;51(No. 5):818-23.

[5] Ishihara JY, Terra MH. On the Lyapunov theorem for singular systems. IEEE Trans Automat Contr 2002;47(No. 11): 1926-30.

[6] Lei Z, Ho DW, Zhai G. Stability analysis of switched linear singular systems. Automatica 2013;49(No. 5):1481-7.

[7] Sastry S, Desoer CA. Jump behavior of circuits and systems. IEEE Trans Circuits Syst Dec. 1981;28:1109-24.

[8] J Li G, Lie TT, Soh CB, H Yang G. "Decentralized $\mathrm{H}_{\infty}$ control for power system stability enhancement. Int J Elect Power Energy Syst Oct. 1998;20(No. 7):453-64.

[9] Zaghdoud R, Salhi S, Ksouri M. Stabilization problem of singular systems via proportional plus derivative state feedback. Proceed Int Conf Contr Eng Inf Technol 2013;2: 6-9.

[10] Chang WJ, Hsu FL. Sliding mode fuzzy control for TakagiSugeno fuzzy systems with bilinear consequent part subject to multiple constraints. Inf Sci 2016;327:258-71.

[11] Chang WJ, Chen PH, Ku CC. Variance and passivity constrained sliding mode fuzzy control for continuous stochastic nonlinear systems. Neurocomputing 2016;201:29-39.

[12] Chang WJ, Hsu FL, Ku CC. Complex performance control using sliding mode fuzzy approach for discrete-time nonlinear systems via T-S fuzzy model with bilinear consequent part. Int J Contr Automat Syst 2017;15(No. 4): 1901-15.

[13] Chang WJ, Qiao HY, Ku CC. Sliding mode fuzzy control for nonlinear stochastic systems subject to pole assignment and variance constraint. Inf Sci 2018;432:133-45.

[14] Chadli M, Karimi HR, Shi P. On stability and stabilization of singular uncertain Takagi-Sugeno fuzzy systems. J Franklin Inst 2014;351(No. 3):1453-63.

[15] Huang CP. Stability analysis of discrete singular fuzzy systems. Fuzzy Sets Syst 2005;151(No. 1):155-65.

[16] Ren H, Karimi HR, Lu R, Wu Y. Synchronization of Network Systems via Aperiodic Sampled-Data Control With Constant Delay and Application to Unmanned Ground Vehicles. IEEE Trans Ind Electron 2019;67(No. 6):4980-90.

[17] Ren H, Lu R, Xiong J, Wu Y, Shi P. Optimal Filtered and Smoothed Estimators for Discrete-Time Linear Systems with Multiple Packet Dropouts Under Markovian Communication Constraints. IEEE Trans Cybern 2019:1-13.

[18] Tseng CL, Wang SY, Lin SC, Chen YY. "Interval type-2 Takagi-Sugeno fuzzy controller design for a class of nonlinear singular networked control systems," International conference on Fuzzy Theory and Its Applications (iFUZZY2012). 2012. p. 268-72.

[19] Willems JC, Trentelman HL. Synthesis of dissipative systems using quadratic differential forms: Part I. IEEE Trans Automat Contr 2002;47(No. 1):53-69.

[20] Trentelman HL, Willems JC. Synthesis of dissipative systems using quadratic differential forms: Part II. IEEE Trans Automat Contr 2002;47(No. 1):70-86.

[21] Chang WJ, Huang BJ, Chen PH. Fuzzy stabilization for nonlinear discrete ship steering stochastic systems subject to state variance and passivity constraints. Math Probl Eng 2014:1-12.

[22] Chang WJ, Ku CC, Chang W, Huang PH. Passive fuzzy control with relaxed conditions for discrete affine T-S fuzzy systems. Int J Innovat Comput Inf Contr 2007;3(No. 4): 853-71.

[23] Zhao F, Zhang Q, Yan X, Cai M. $\mathrm{H}_{\infty}$ filtering for stochastic singular fuzzy systems with time-varying delay. Nonlinear Dynam 2015;79(No. 1):215-28.

[24] Sun S, Ma J. Optimal filtering and smoothing for discretetime stochastic singular systems. Signal Process 2007; 87(No.1):189-201. 
[25] Abdelaziz THS, Valášek M. Direct algorithm for pole placement by state-derivative feedback for multi-input linear systems-nonsingular case. Kybernetika 2005;41(No. 5): 637-60.

[26] Cardim R, Teixeira MCM, Assunção E, Faria FA. Control designs for linear systems using state-derivative feedback. Struct Contr 2008:1-28.

[27] Abdelaziz THS, Valášek M. Pole-placement for SISO linear systems by state-derivative feedback. IEE Proc Contr Theory Appl 2004;151(No. 4):377-85.

[28] Duan YF, Ni YQ, Ko JM. State-derivative feedback control of cable vibration using semiactive magnetorheological dampers. Computer-Aided Civil Infrastruct Eng 2005;20(No. 6):431-49.

[29] Reithmeier E, Leitmann G. Robust vibration control of dynamical systems based on the derivative of the state. Arch Appl Mech 2003;72(No. 11-12):856-64.

[30] Kwak SK, Washington G, Yedavalli RK. Acceleration feedback based active and passive vibration control of landing gear components. J Aerospace Eng 2002;15(No. 1):1-9.
[31] Chang WJ, Shih YJ. Fuzzy control of multiplicative noised nonlinear systems subject to actuator saturation and $\mathrm{H}_{\infty}$ performance constraints. Neurocomputing 2015;148: 512-20.

[32] Chang WJ, Chen PH, Ku CC. Mixed sliding mode fuzzy control for discrete-time nonlinear stochastic systems subject to variance and passivity constraints. IET Contr Theory Appl 2015;9(No. 16):2369-76.

[33] Taniguchi T, Tanaka K, Wang HO. Fuzzy descriptor systems and nonlinear model following control. IEEE Trans Fuzzy Syst 2000;8(No. 4):442-52.

[34] Boyd S, Ghaoui LE, Feron E, Balakrishnan V. Linear Matrix Inequalities in System and Control Theory. Philadelphia, PA USA: SIAM; 1994.

[35] Wang G, Bo H. Stabilization of singular Markovian jump systems by generally observer-based controllers. Asian J Contr 2016;18(No. 1):328-39.

[36] Bai J, Liu X, Lu R, Shu J. "D stabilization of the fuzzy singular systems," The 34th Chinese Control Conference. Hangzhou, China: CCC); 2015. p. 3653-7. 